Research Article

\title{
Parameter Resolution of the Estimation Methods for Power Law Indices
}

\author{
Zheng-Yun Zhou and Yi-Ming Ding \\ School of Science, Wuhan University of Technology, Wuhan 430070, China \\ Correspondence should be addressed to Yi-Ming Ding; dingym@whut.edu.cn
}

Received 26 February 2021; Accepted 16 June 2021; Published 3 July 2021

Academic Editor: Ruguo Fan

Copyright (c) 2021 Zheng-Yun Zhou and Yi-Ming Ding. This is an open access article distributed under the Creative Commons Attribution License, which permits unrestricted use, distribution, and reproduction in any medium, provided the original work is properly cited.

\begin{abstract}
The accuracy of parameter estimation plays an important role in economic and social models and experiments. Parameter resolution is the capability of an estimation algorithm to distinguish different parameters effectively under given noise level, which can be used to select appropriate algorithm for experimental or empirical data. We use a flexible distinguishing criterion and present a framework to compute the parameter resolution by bootstrap and simulation, which can be used in different models and algorithms, even for non-Gaussian noises. The parameter resolutions are computed for power law models and corresponding algorithms. For power law signal, with the increase of SNR, parameter resolution is finer; with the decrease of parameter, the resolution is finer. The standard deviation of noise and parameter resolution satisfies the linear relation; it relates to interval estimation naturally if the estimation algorithm is asymptotically normal. For power law distribution, parameter and resolution satisfy the linear relation, and experimental slope and theoretical slope tend to be consistent when significance level approaches zero. Last, we select an algorithm with finer resolution to estimate the Pareto index for the Forbes list of global rich data in recent 10 years and analyze the changes in the gap between the rich and the poor.
\end{abstract}

\section{Introduction}

The processing of economic and social experimental data requires accurate parameter estimation [1-4], which can analyze the experimental results more precisely [5-7]. Parameter estimation in physical model is an important part of physical analysis $[8,9]$.

In order to measure the accuracy of parameter estimation, we use parameter resolution $[10,11]$ which was considered in [12] for EM algorithm and estimation algorithms for linear regression model [13] before. Lu and Yu carried out a preliminary study on the resolution of parameters and analyzed the EM algorithm [12]. Focusing on the linear model, Zhang and Yu [13] further studied the evaluation criteria of parameter resolution. In order to investigate the parameter resolution further, this paper takes the power law model as an example to explore its influencing factors and practical applications. Parameter resolution is the capability of an estimation algorithm to distinguish different parameters effectively under given noise level, which can be used to select appropriate algorithm for experimental or empirical data. In this paper, we investigate parameter resolution systematically and present a framework to compute the parameter resolution by bootstrap and simulation based on classification rather than clustering $[12,13]$. Our framework can be used in different models and algorithms, even for non-Gaussian noises. We use power law models, corresponding algorithms, and real data (Forbes rich list) as prototypes to show that the parameter resolution is always computable; it relates to interval estimation in statistics naturally if the estimation is asymptotically normal, but the situation of complicated noise is different.

In fact, the parameter identification is closely related to the selection of model, algorithm, and distinguishing standard. For the same set of experimental data, different models or the same model chooses different algorithms, and the estimated parameters may not be consistent, which makes the prediction capability of the unknown data 
different. Hence, the selection of the model and algorithm is important in the data analysis. The model and algorithm can be evaluated from the following four aspects: accuracy, robustness, calculation speed, and simplicity of the model. This paper puts forward the concept of parameter resolution, which is used to evaluate the accuracy of model and algorithm.

In this paper, the power-law model is selected, and the parameter resolution is studied by using nonlinear leastsquare method and maximum likelihood method. In order to study the relationships between the parameter resolution and the influencing factors, this paper selects different discrimination methods, estimation algorithms, parameter values, and noise types. The research shows that the classification algorithm has lower error rate, and lsqcurvefit algorithm can distinguish two more similar signals; with the increase of parameter values, the accuracy of parameter resolution is lower. In other words, the similar signals are more difficult to be separated, and the absolute error of local parameters is small; it is found that when the parameter estimation is asymptotically normal, the parameter resolution can be predicted by interval estimation, and other cases can also be obtained by experimental calculation; the accuracy of parameter resolution increases with the increase of signal-to-noise ratio and decreases with the increase of noise intensity. By changing the significance level, the theoretical and experimental values are compared, and the feasibility of using interval estimation to calculate parameter resolution is verified.

\section{Parameter Resolution}

In general, the parameter resolution of an algorithm depends on model, noise, and sample size as well as the criterion to distinguish two close signals. The distinguishing criterion is the most important part; we will use a flexible criterion which can be adjusted by two parameters, $\alpha$ (precision) and $\beta$ (robustness).

Consider the model

$$
y_{\theta}(t)=f(t ; \theta)+\varepsilon,
$$

where $f(t ; \theta)$ is the signal containing information of some physical process and $\varepsilon$ is a zero mean independent identically distributed (IID) noise. The strength of noise is measured by the signal-to-noise ratio (SNR). If we have a sample $\left\{\left(y_{i}, t_{i}\right)\right\}(i=1, \ldots, N)$ for some $\theta$, then SNR $=10 \lg \left(P_{\text {signal }} /\right.$ $\left.P_{\text {noise }}\right)$, where $P_{\text {signal }}=\sum_{i=1}^{n}\left[f\left(t_{i} ; \theta\right)\right]^{2} / n$ and $P_{\text {noise }}=\sum_{i=1}^{n}$ $\varepsilon_{i}^{2} / n$ are the mean intensities of signal and noise, respectively. Select an algorithm $\mathbf{A}$ to obtain an estimation of $\theta$; how can we say that algorithm $\mathbf{A}$ distinguishes two close parameters $\theta_{1}$ and $\theta_{2}$ effectively for fixed noise level and sample size? Such a problem can be resolved by bootstrap and simulation; we provide the following distinguishing criterion.

\subsection{Distinguishing Criterion}

(1) Generate two groups of $m(=100)$ signals for given noise level and sample size, and use algorithm $\mathbf{A}$ to obtain estimations $\hat{\theta}=\left\{\theta_{11}, \ldots, \theta_{1 m}, \theta_{21}, \ldots, \theta_{2 m}\right\}$.

(2) Divide the elements in $\widehat{\theta}$ into two groups by distinguishing method $\mathbf{B}$, and compute the misjudgement rate $m$ or the precision $1-m$. If $m$ is less than a prescribed threshold $\alpha$ (e.g., $\alpha=0.10$ ), algorithm $\mathbf{A}$ can distinguish the two signals under significance level $\alpha$ successfully.

(3) Repeat 1 and 2 for $k$ times; if the success rate is greater than a prescribed threshold $\beta$ (e.g., $\beta=0.90$ ), then algorithm $\mathbf{A}$ can distinguish the two signals effectively under significance level $\alpha$ and robustness $\beta$.

In the second step, to divide the elements in $\widehat{\theta}$ into two groups, one can use clustering $[9,10]$, which is an unsupervised learning method. We shall adopt classification, a supervised learning method, because it can lead to more precise parameter resolution (see Figure 1(a)). We use the significance level $\alpha$ to adjust the precision of each bootstrap simulation and $\beta$ to control the robustness of all the simulations. The introduction of $\alpha$ and $\beta$ makes the distinction criteria for similar signals and distributions more flexible. If $\alpha$ and $\beta$ are fixed, the distinguishing criterion is also fixed. The parameter resolution of the estimation algorithm $\mathbf{A}$ is the minimal interval between two parameters so that $\mathbf{A}$ can distinguish them effectively.

For model (1), given a distinguishing criterion and a noise, the parameter resolution of algorithm $\mathbf{A}$ at $\theta_{0}$ is the minimal number $\operatorname{PR}_{\mathbf{A}}\left(\theta_{0}\right)$ so that if $\left|\theta_{0}-\theta\right|>\operatorname{PR}\left(\theta_{0}\right)$ then $\mathbf{A}$ can effectively distinguish the two signals $y_{\theta_{0}}(t)$ and $y_{\theta_{1}}(t)$. In other words, if $\theta_{1}$ belongs to the ambiguous interval

$$
\left(\theta_{0}-\mathrm{PR}_{\mathbf{A}}\left(\theta_{0}\right), \theta_{0}+\mathrm{PR}_{\mathbf{A}}\left(\theta_{0}\right)\right)
$$

the two signals $y_{\theta_{0}}(t)$ and $y_{\theta_{1}}(t)$ cannot be effectively distinguished by $\mathbf{A}$. In general, the ambiguous interval may not be symmetric, but it is almost symmetric (especially when the noise is symmetric), and we assume that it is symmetric for briefness. Using $\mathrm{PR}_{\mathbf{A}}\left(\theta_{0}\right)$, we can obtain the absolute error, $\operatorname{AE}\left(\theta_{0}\right)=\operatorname{PR}_{\mathbf{A}}\left(\theta_{0}\right)$, and the relative error, $\operatorname{RE}\left(\theta_{0}\right)=\operatorname{PR}_{\mathbf{A}}\left(\theta_{0}\right) / \theta_{0}$.

The steps of computing parameter resolution $\mathrm{PR}_{\mathbf{A}}\left(\theta_{1}\right)$ for an algorithm $\mathbf{A}$ at $\theta=\theta_{1}$ are formulated in the following experimental procedures.

\subsection{Experimental Procedures for Algorithm $\boldsymbol{A}$}

(1) Distinguishing Criterion. Select appropriate distinguishing criterion, $\alpha$ and $\beta$

(2) Produce two noisy signals $y_{1}=f\left(t_{i} ; \theta_{1}\right)+\varepsilon_{1}$ and $y_{2}=f\left(t_{i} ; \theta_{2}\right)+\varepsilon_{2}, i=1, \ldots, N$ 


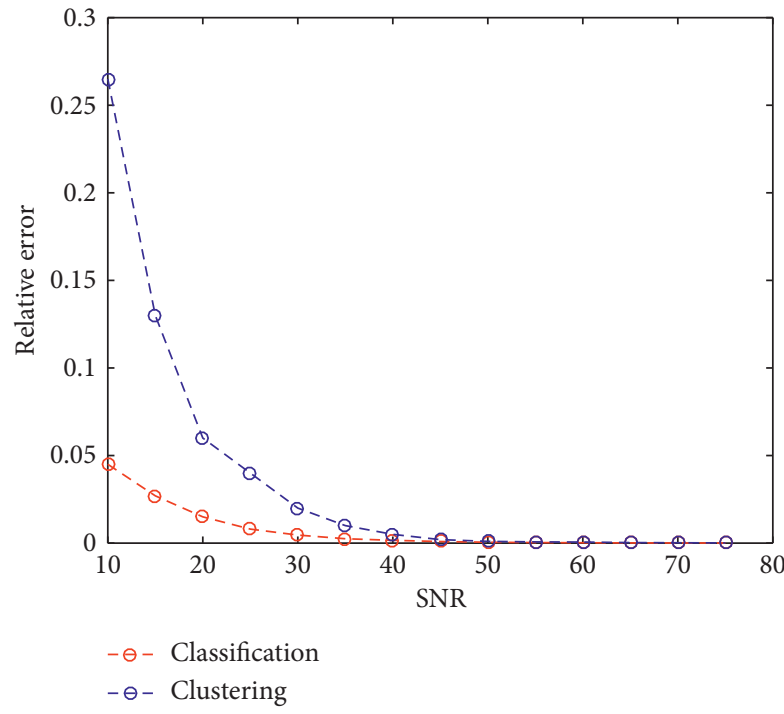

(a)

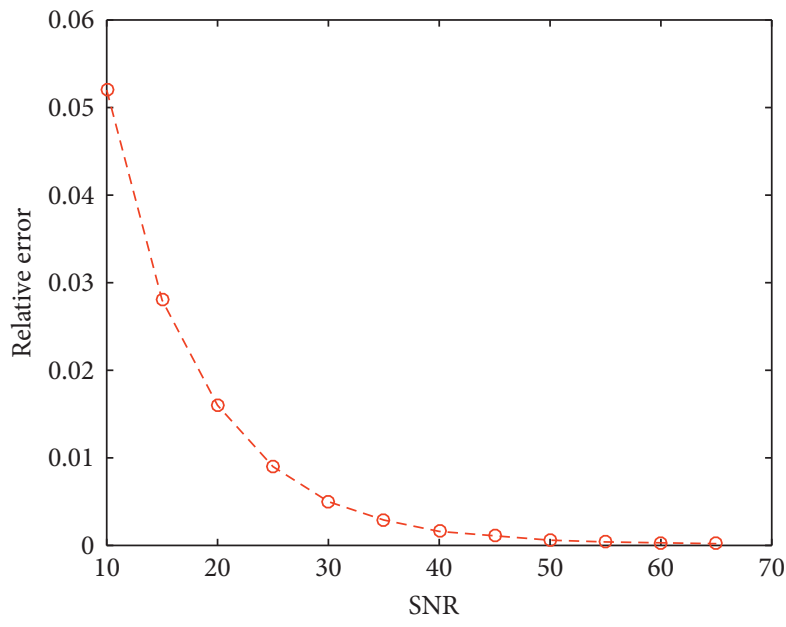

(c)

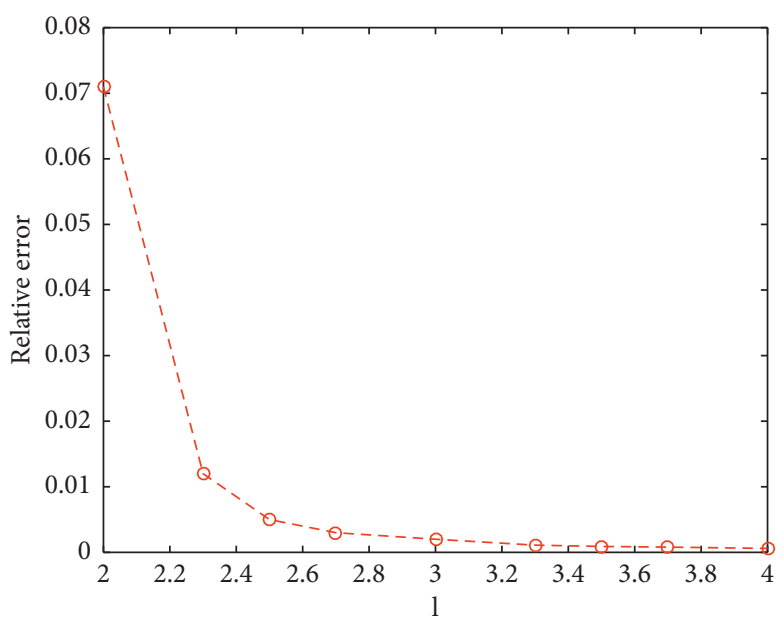

(b)

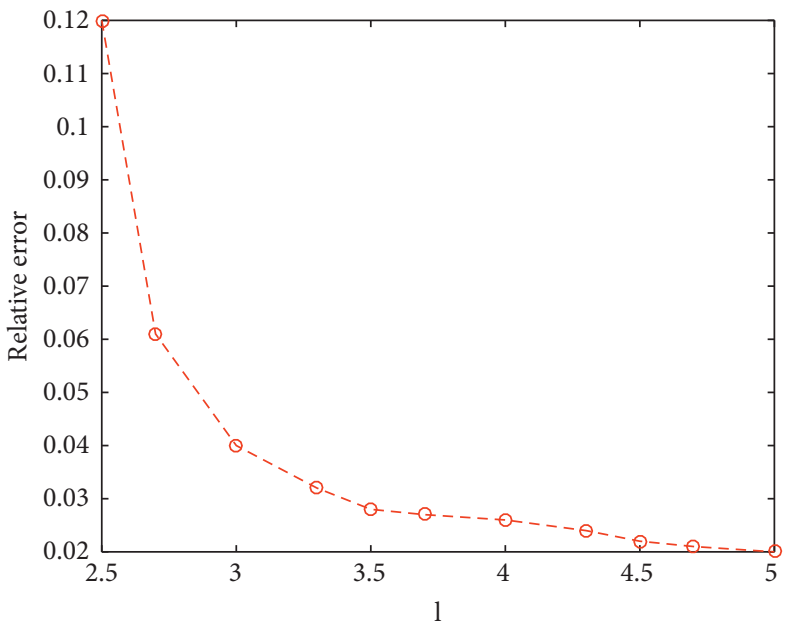

(d)

FIGURE 1: Parameter resolution can be used in different models and algorithms, even for non-Gaussian noises. (a) The relationship between relative error and SNR with different distinguishing method of linear model. The estimation algorithm is the least-squares method. (b) The relative error under different power law noise of the linear model. The estimation algorithm is the least-squares method. (c) The relative error under different SNR of the exponential model. The estimation algorithm is the nonlinear least-squares method. (d) The relative error under different power law noise of the exponential model. The estimation algorithm is the nonlinear least-squares method.

(3) Estimation. Generate $n$ groups of parameter estimations $\theta_{1}^{(1)}, \ldots, \theta_{1}^{(n)}$ and $\theta_{2}^{(1)}, \ldots, \theta_{2}^{(n)}$ by algorithm A

(4) Distinguishing. Determine whether two signals are effectively distinguished by the selected distinguishing criterion

(5) Calculation. Adjust $\theta_{2}$ to reduce $\left|\theta_{1}-\theta_{2}\right|$ until two signals cannot be distinguished effectively by the distinguishing criterion, and obtain the minimum distinguished interval which is the desired parameter resolution

Next, we will choose linear and exponential models as examples to show that parameter resolution can be applied to each model, as is shown in Figure 1. Figure 1(a) is compared with the experiment in [9]; it shows that the parameter resolution of classification is better than that of clustering. The estimation algorithm of (a) and (b) is the least-square method. Figures $1(\mathrm{c})$ and $1(\mathrm{~d})$ are both exponential models but under different noise type.

Set the following in the experimental procedures: (a) $y_{1}=a_{0}+a_{1} x+\varepsilon_{1}$ and $y_{2}=a_{0}+a_{2} x+\varepsilon_{2} . t=0.01: 0.01$ : $10, a_{0}=0.09, a_{1}=10, \varepsilon_{1}$ and $\varepsilon_{2}$ are independent Gaussian noise with zero mean, and SNR $\in[10,75]$. Estimation algorithm is nonlinear least-squares method. Distinguishing methods are Support Vector Machine (SVM) classification and peak-based clustering [14]; $\alpha=0.1$ and $\beta=0.9$. (b) The model is the same as (a). For power law noise, $\varepsilon=x_{\min }(1-r)^{(1 / 1-l)}$, with $r$ subject to uniform distribution $[0,1], x_{\min }=0.01$, and $l \in[2,4]$. (c) The model is as follows: 
$y=a_{1} \cdot e^{-a_{2} t}+\varepsilon, a_{1}=5, a_{2}=10$, and SNR $\in[10,75]$. (d) The model is the same as (c). For power law noise, $\varepsilon=x_{\min }(1-r)^{(1 / 1-l)}$, with $r$ subject to uniform distribution $[0,1], x_{\min }=0.01$, and $l \in[2.5,5]$.

Power law distribution exists in many fields of nature and daily life [15-19]. For example, the distribution of the urban population's size, the magnitude of earthquake [20], size of interplanetary debris [21], the distribution of the moon pit diameter on the surface of the moon [22], distribution of the scale of the war [23], and distribution of the personal wealth are all subject to the power law distribution. There are various power law distribution phenomena in nature and social life, and the study of them is of great significance $[14,24-30]$. Accurate estimation of power law indices is important, because different indices lead to different explanation. Power law signal and power law distribution are two forms of power law models. We investigate parameter resolution of the estimation methods for indices of power law models.

\section{Power Law Signal}

3.1. Compare Distinguishing Methods and Algorithms. Parameter of power law signal can be estimated by two algorithms: lsqcurvefit and nlinfit. Two estimated algorithms linearize the nonlinear function via the first-order term of its Taylor series for the use of linear least squares. Initial value of lsqcurvefit algorithm does not affect the iteration result; it affects the iteration speed. Initial value of nlinfit algorithm is more sensitive. Parameter of power law distribution can be estimated by maximum likelihood method. We will compute the parameter resolutions of these algorithms by the experimental procedures and reveal the properties of parameter resolution.

At first, we compare the effect of distinguishing methods (clustering and classification) on parameter resolution. Set the following in experimental procedures: $y_{1}=a_{0} x^{-a_{1}}+\varepsilon_{1}$ and $y_{2}=a_{0} x^{-a_{2}}+\varepsilon_{2} . t=0.01: 0.01: 3$ (uniform in logarithm), $x=10^{t}, a_{0}=5, a_{1}=10$, and $\varepsilon_{1}, \varepsilon_{2}$ are independent Gaussian noise with zero mean and variance equal to $P_{\text {noise }}, P_{\text {noise }} \in\left[10^{-9}, 10^{-3}\right]$. Algorithms are lsqcurvefit and nlinfit. Distinguishing methods are Support Vector Machine (SVM) classification and peak-based clustering [31]; $\alpha=0.1$ and $\beta=0.9$.

Figure 2 shows the parameter resolution under different noise levels by using different distinguishing methods (clustering and classification) and algorithms (lsqcurvefit and nlinfit). As is shown in Figure 2(a), by comparing the differences of parameter resolution between different distinguishing methods, it can be observed that the number of misjudgements in using classification algorithm is less than that of clustering, and the parameter resolution is more precise. Because classification is supervised learning, objects can be tagged according to certain standards and then classified according to labels. Clustering aggregates samples without their own categories into different groups. Therefore, classification is suitable for situations where categories or classification systems have been determined, and clustering is suitable for situations where there is no classification system, and the number of categories is uncertain. For the two parameters determined by the category, the accuracy of classification is more precise because $\mathrm{AE}$ is smaller. Because of the locality of parameter estimation, when parameter is small, estimation is more accurate, and the parameter resolution is better. We can also find that the parameter resolution of lsqcurvefit algorithm is finer.

From Figure 2(b), it is observed that there is a linear relationship between the absolute error and the standard deviation $\sigma$. Fitting by linear model via least square of the line with SVM classification and lsqcurvefit algorithm, we obtain

$$
\mathrm{AE}=8.397 \sigma+0.0023
$$

with $R^{2}=0.9946$. It indicates that there is a positive linear relationship between the standard deviation of noise and parameter resolution.

3.2. Influence of Noise Type. Now, we investigate the influence of noise type on parameter resolution. We fix the SVM classification and lsqcurvefit algorithm to compare the parameter resolution under IID Gaussian noise and IID power law noise. Set the following values in experimental procedures: $t=0.01$ : $0.01: 3, x=10^{t}, a_{0}=5 . \alpha=0.1$, and $\beta=0.9$. (a) $a_{1}=1: 3$, Gaussian noise: $P_{\text {noise }}=3 \times 10^{-7}$, the power law noise admits the following density, $p(s)=C|s|^{-2}$, and $|s| \geq 0.1$. (b) $a_{1}=1: 10$, and the others are the same as (a). (c) $a_{1}=10 ; 0<\mathrm{SNR}<40$. (d) $a_{1}=10 ; 1 \times 10^{-9}<P_{\text {noise }}<1$ $\times 10^{-3}$.

The results are presented in Figure 3. From Figure 3(a) and the red line in Figure 3(b), the absolute error (AE) is varied at different power law indices. If the noise is changed to power law, the relationship between $\mathrm{AE}$ and the power law index is shown with blue lines in Figures 3(a) and 3(b), which indicates stronger growth with index. It implies that parameter resolution of power law model depends on specific power law index, which is different from linear regression model [10]. The changes of relative error under different SNR and $P_{\text {noise }}$ are shown in Figures 3(c) and 3(d). We can find that, with the increase of SNR, parameter resolution decreases; with the increase of $P_{\text {noise }}$, parameter resolution increases. Figure 3 indicates that the parameter resolutions are quite different when the perturbation noise is changed.

\section{Power Law Distribution}

4.1. Experimental Procedure. In order to study the parameter resolution of maximum likelihood estimation for power law distribution, according to experimental procedure, we select the following parameters to compute the parameter resolution of power law distribution: produce two power law distributions: $\quad x_{1}=x_{\min }(1-r)^{\left(1 / 1-a_{1}\right)}$ and $x_{2}=x_{\min }$ $(1-r)^{\left(1 / 1-a_{2}\right)}$, with random numbers $r$ obeying the uniform distribution in $[0,1]$; estimated algorithm is maximum likelihood method, data size is 500, 1000, and 2000, respectively, and the results are shown in Figure 4(a). 


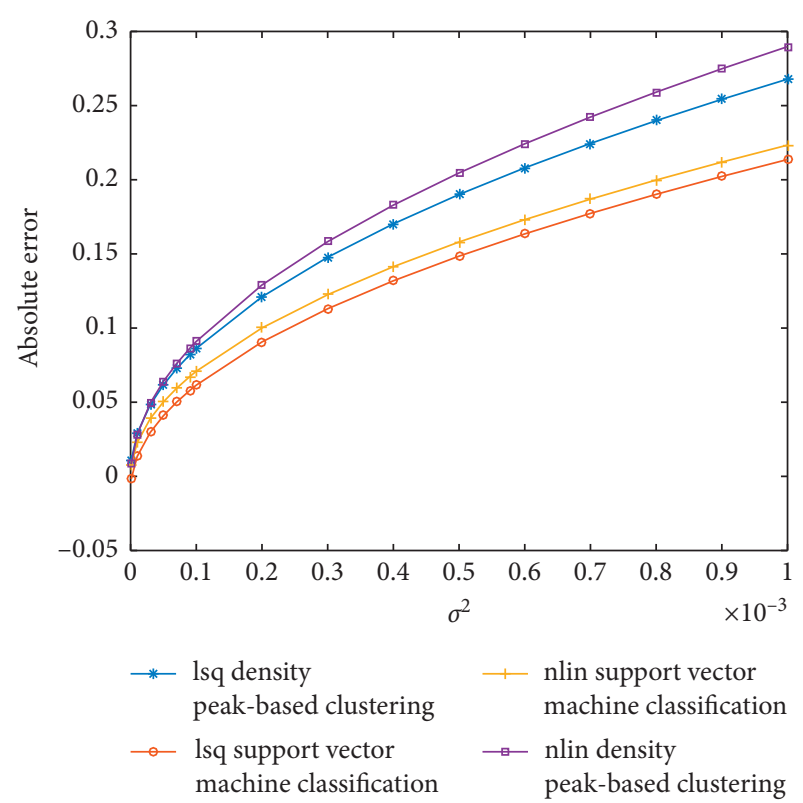

(a)

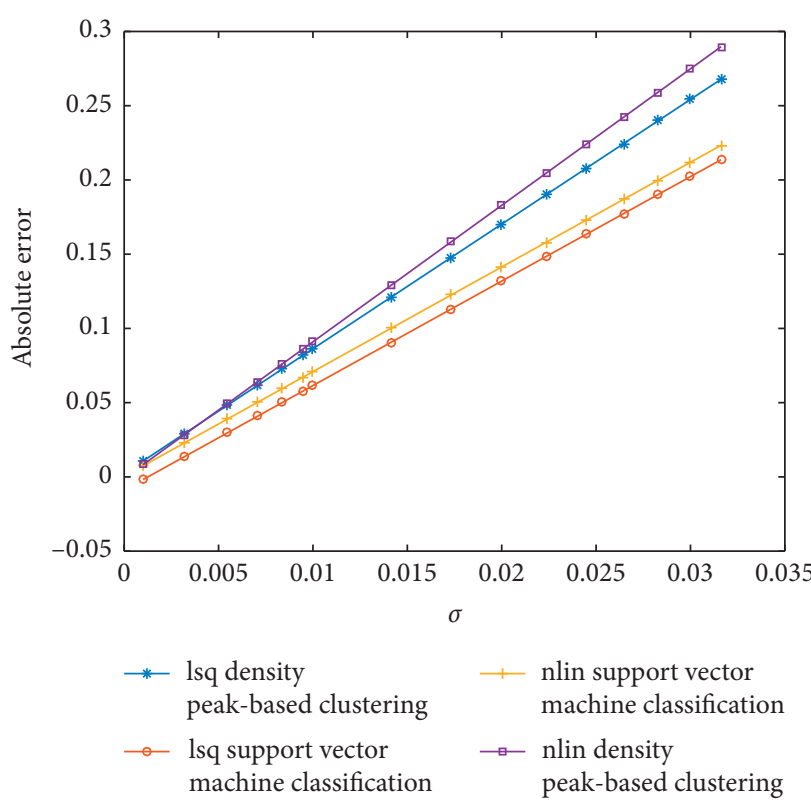

(b)

Figure 2: Purple, blue, yellow, and red lines, respectively, correspond to the method of nlinfit algorithm with density peak-based clustering, lsqcurvefit algorithm with density peak-based clustering, nlinfit algorithm with SVM classification, and lsqcurvefit algorithm with SVM classification; $\alpha=0.1$ and $\beta=0.9$. (a) The relationship between absolute error and variance $\sigma^{2}$. (b) The relationship between parameter resolution and $\sigma$.

4.2. Experimental Results and Analysis. It can be seen from Figure 3(a) that there is an obvious linear relationship between absolute error and power index. For sample size $n=2000$, the curve is fitted to be a linear equation $y=$ $0.0685 a-0.0641$ with $R^{2}=0.9915$. This indicates that the absolute error can be approximated by the standard deviation of noise.

In the power law model, the parameter resolution based on absolute error and parameter $a$ satisfies the positive linear relationship:

$$
\mathrm{AE}=\frac{c_{0}+b(a-1)}{\sqrt{n}}
$$

where $a$ is the power law index and $n$ is the sample size.

In fact, the maximum likelihood estimation for different parameters $a_{1}$ and $a_{2}$ in power law distribution is carried out [32]:

$$
\begin{aligned}
& \widehat{a}_{1} \sim N\left(a_{1}, \frac{\left(a_{1}-1\right)^{2}}{n}\right), \\
& \widehat{a}_{2} \sim N\left(a_{2}, \frac{\left(a_{2}-1\right)^{2}}{n}\right) .
\end{aligned}
$$

The regions where two normal distribution overlap may be misjudgement. The confidence interval at significance level $\alpha$ of $\hat{a}_{1}$ can be calculated via the pivot amount $u=\left(\widehat{a}_{1}-a_{1} /(a-1) / \sqrt{n}\right) \sim N(0,1)$.

$$
\left[a_{1}-\frac{a_{1}-1}{\sqrt{n}} U_{1-(\alpha / 2)}, a_{1}+\frac{a_{1}-1}{\sqrt{n}} U_{1-(\alpha / 2)}\right],
$$

where $U_{1-(\alpha / 2)}$ is quantile of normal distribution. The length of confidence interval is $2 \cdot\left(a_{1}-1 / \sqrt{n}\right) U_{1-(\alpha / 2)}$. The length of confidence interval and the ambiguous interval are almost identical when $\alpha \approx 0$ and $\beta \approx 1$. The parameters resolution based on absolute error meets the linear relationship if confidence level $1-\alpha$ and the sample size $n$ are fixed, with theoretical slope $2 \cdot\left(U_{1-(\alpha / 2)} / \sqrt{n}\right)$. Equation (3) can be proved in the same way, because $\widehat{a}_{1}$ and $\widehat{a}_{2}$ are normally distributed [33]. This indicates that if the estimation is asymptotically normal, it can be approximated by interval estimation. Other noise conditions do not have such a rule, but parameter resolution is always computable.

Parameter resolution is related to distinguishing criterion, especially the significance level $\alpha$ and robustness $\beta$. Fix $\beta=0.9$, and $\alpha$ varies from 0.05 to 0.20 ; the slope of absolute error and experimental slope are obtained. Details are shown in Figure 4(b). The theoretical slope is greater than experimental slope because the parameter resolution in the worst case is obtained by the theory; that is, there may be no points in the shadow part. Figure 4(b) shows that, with the increase of $\alpha$, the experimental slope and theoretical slope decrease, and absolute error will decrease, which means that we can distinguish two similar signals more easily. The difference between experimental slope and theoretical slope increases gradually; it tends to be consistent when $\alpha \longrightarrow 0$. Figure 4(b) also shows the accuracy of interval estimation.

\section{Application}

Forbes magazine publishes an annual list of the richest people. The list includes the net worth of each individual as 


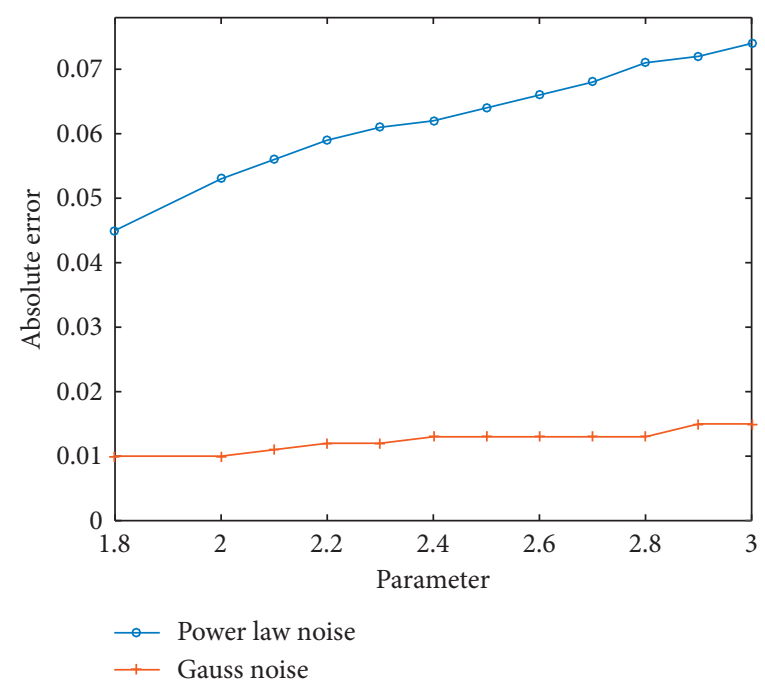

(a)

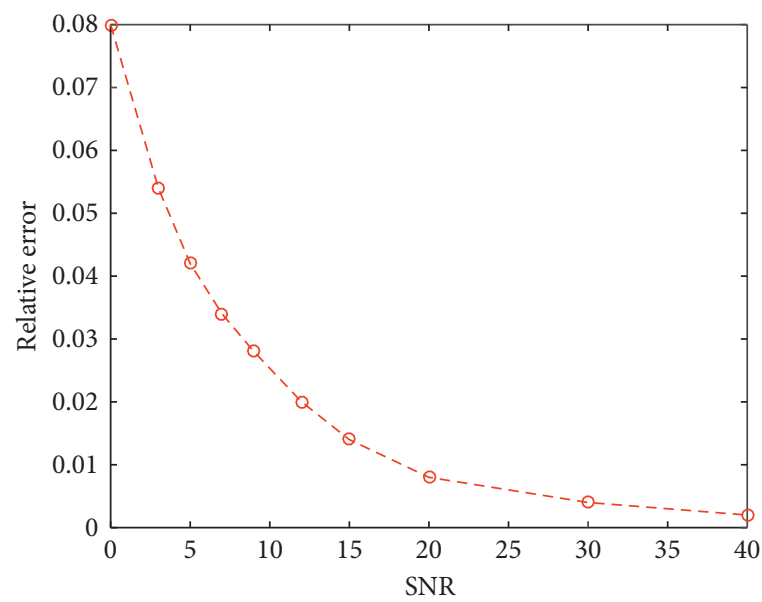

(c)

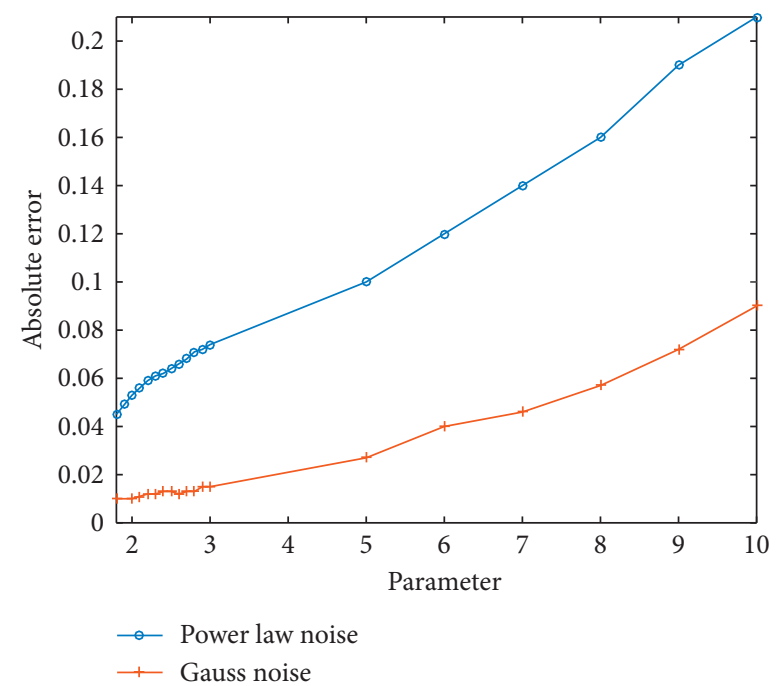

(b)

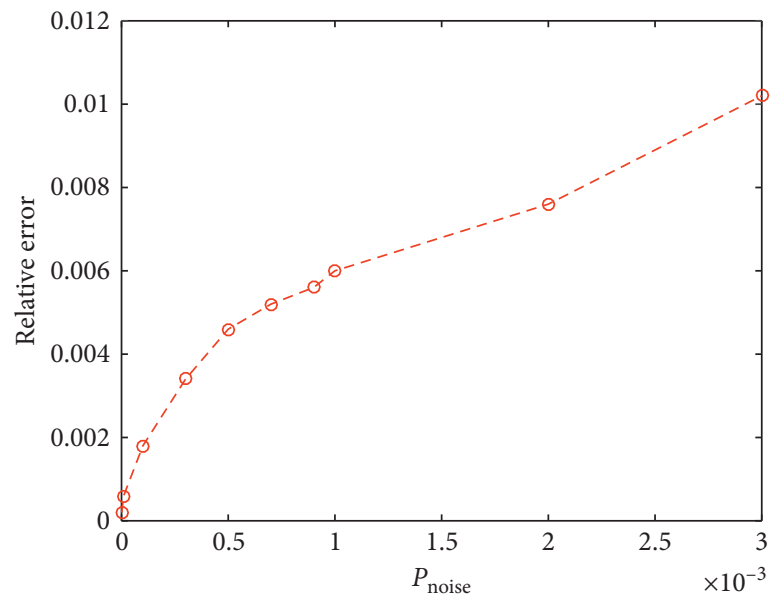

(d)

Figure 3: The parameter resolution of lsqcurvefit at different power law index, under the perturbation of Gaussian noise and power law noise and the distinguishing criterion in SVM classification; $\alpha=0.1, \beta=0.9$. (a) The relationship between absolute error and power law indexes under the perturbation of Gaussian noise and power law noise. The power law index ranged from 1.8 to 3. (b) The relationship between absolute error and power law indexes under the perturbation of Gaussian noise and power law noise. The power law index ranged from 1.8 to 10 . (c) The relative error under different SNR. (d) The relative error under different $P_{\text {noise }}$.

well as background information about the businesses that have led to this prosperity. It includes individuals involved in all sectors of the economy, such as computer software, financial investments, retailing, and computer hardware as well as media, entertainment, communication, real estate, and many other sectors.

Although the people included in the Forbes 400 list made their fortunes in various different ways, the distribution of their wealth exhibits a striking statistical regularity. This regularity describes not only the wealth distribution at the very top of wealth range but also provides insights into any order of magnitude below this range of wealth distribution. These empirical findings can clarify the nature of the wealth accumulation process and its relationship to central issues such as market efficiency and market volatility.

Wealth distribution is typically studied by employing data about the number of individuals within each wealth range (e.g., the number of individuals with wealth between $\$ 100,000$ and $\$ 150,000)$. Here, we employ a different approach; we use the Forbes 400 list to study the distribution of wealth at the top wealth range. The advantage of this approach is to use very specific data about the wealth of everyone on the list, as well as avoiding the aggregation problems of standard approach. The disadvantage, of course, is that this approach is limited to the very top of the wealth distribution. However, as we argue below, the results obtained with this approach may shed light on a much wider range of the wealth scale and on the capital investment process in general $[34,35]$.

According to top 100 Forbes global list from 2012 to 2020 , this paper counts the trade's distribution each year, and the results are shown in Table 1. We divide the trade into four categories: finance, IT, industry (retail, manufacturing, and mining), and service. In general, the overall distribution 


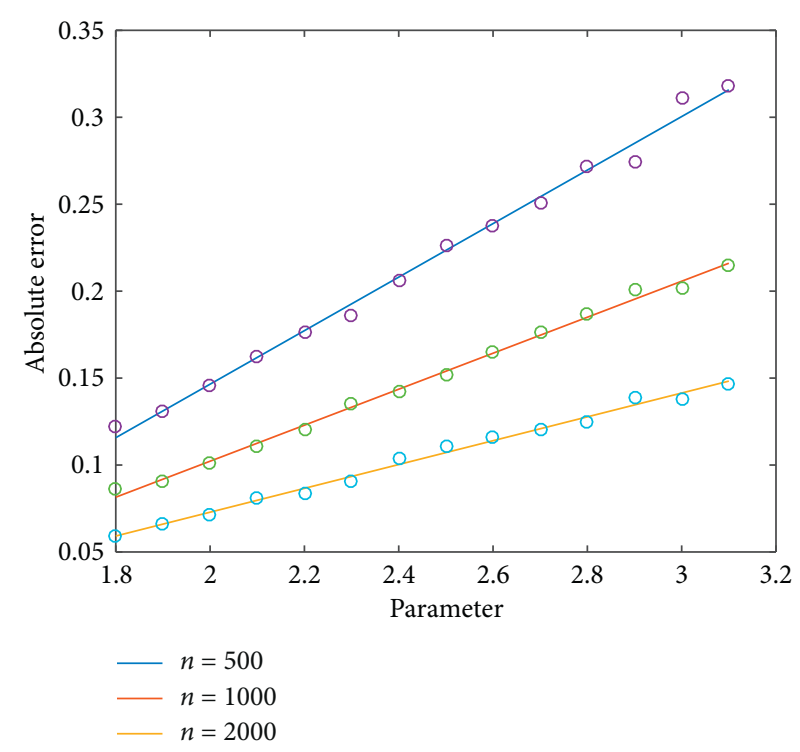

(a)

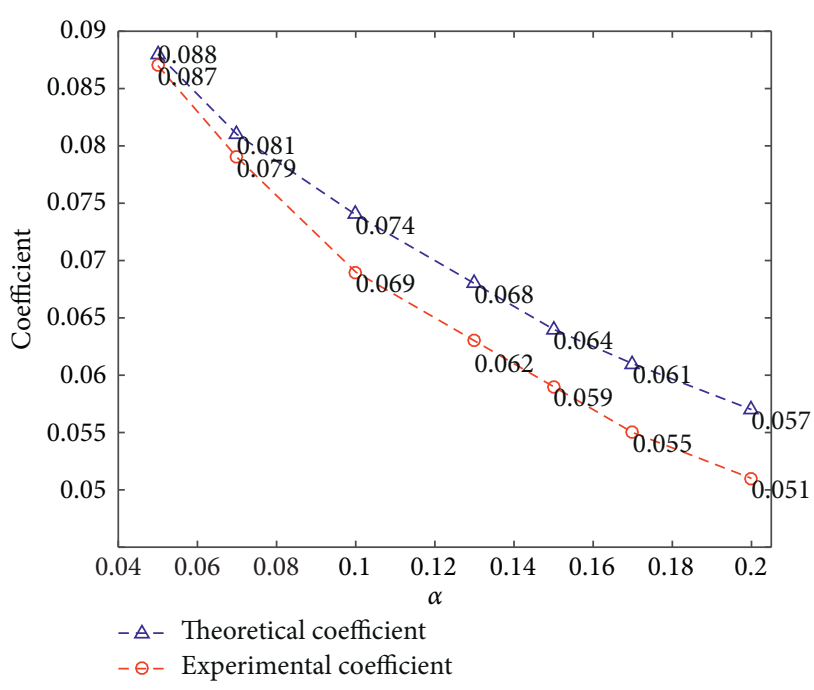

(b)

FIGURE 4: The parameter resolution of the maximum likelihood method. The distinguishing criterion in SVM classification; $\alpha=0.1, \beta=0.9$. (a) The relationship between absolute error and parameter. Blue, red, and yellow lines correspond to the sample size $n=500,1000,2000$. (b) The laws of theoretical and experimental slope under different $\alpha$. Red and blue lines correspond to theoretical slope and experimental slope.

TABLE 1: Number of every trade per year.

\begin{tabular}{lcccc}
\hline & Finance & IT & Industry & Service \\
\hline 2012 & 19 & 17 & 59 & 5 \\
2013 & 15 & 14 & 63 & 8 \\
2014 & 13 & 15 & 57 & 5 \\
2015 & 18 & 21 & 55 & 6 \\
2016 & 24 & 17 & 56 & 3 \\
2017 & 21 & 16 & 57 & 6 \\
2018 & 21 & 15 & 58 & 6 \\
2019 & 21 & 18 & 58 & 3 \\
2020 & 19 & 17 & 59 & 5 \\
\hline
\end{tabular}

of trades remained unchanged, the number of industries has risen steadily in the past six years, the number of financial sectors has also increased, and the other two fields slightly decreased.

The empirical results show that the distribution of wealth is subject to power law distribution [36-38]. Render shows that if the distribution of wealth follows the power law distribution, the value of personal wealth $\omega_{r}$ and its wealth ranking $r$ are satisfied [39]:

$$
\omega_{r}=a_{0} r^{-a_{1}},
$$

where $r$ is the rank ( $r=1$ being the wealthiest person, etc.), $\omega_{r}$ is the wealth of individual with rank $r, a_{0}$ is a constant, and exponent $a_{1}$ is related to the Pareto exponent, $p$, as $a_{1}=$ $1 / p$ [39]. This implies that when wealth is plotted against rank on a log-log scale, a linear relation is observed for a Pareto distribution. Exponent $a_{1}$ quantifies the level of wealth inequality. As $a_{1}$ decreases, the wealth distribution becomes more uneven, and few individuals have a substantial amount of wealth. A bigger $p$ means a bigger gap between the rich and the poor. We analyze the wealth ranking data from the top 100 Forbes global rich list in the recent 10 years; data can be obtained from https://www. forbes.com.

Determine the original signal $S$ based on the data of 2020 of top 400 Chinese mainland rich. Nonlinear least-squares algorithm is used to fit the signal $S$ according to model (7), and we obtain the fitting values $\widehat{a}_{0}=793.47$ and $\widehat{a}_{1}=0.6097$. Set the following values in experimental procedures: (a) $r=1: 1: 400, a_{0}=793.47, a_{1}=0.6097$, and Gaussian noise: $P_{\text {noise }}=1 \times 10^{-4}$. Distinguishing method is SVM classification, and $(\alpha, \beta)=(0.1,0.9)$. Algorithms are lsqcurvefit and nlinfit, and obtain upper and lower absolute errors, respectively. (b) The Pareto index of top 100 Forbes global rich in the recent 10 years with lsqcurvefit algorithms.

As can be seen from Figure 5(a), the minimum upper and lower bounds of parameters can be distinguished by different algorithms. It indicates that different algorithms will lead to different parameter resolutions. In this condition, the lsqcurvefit algorithm's capability of resolution is better.

In Figure 5(b), we use the top 100 Forbes global rich list in the recent 10 years. According to the experiment, lsqcurvefit algorithm has finer resolution, so we select it to analyze the changes in indices, which are more accurate. The results show the rationality and effectiveness of the parameter resolution in practical application. Noise level is unknown in real life, but when the noise level $1 \times 10^{-9}<P_{\text {noise }}<1 \times 10^{-3}$, it can provide more accurate estimations and lead to better understanding for practical problems. In the recent 10 years, the global gap between the rich and the poor has increased slightly and has been relatively stable in the last two years. The widening of the gap between the rich and the poor is universal in contemporary world and has a certain relationship with economic 


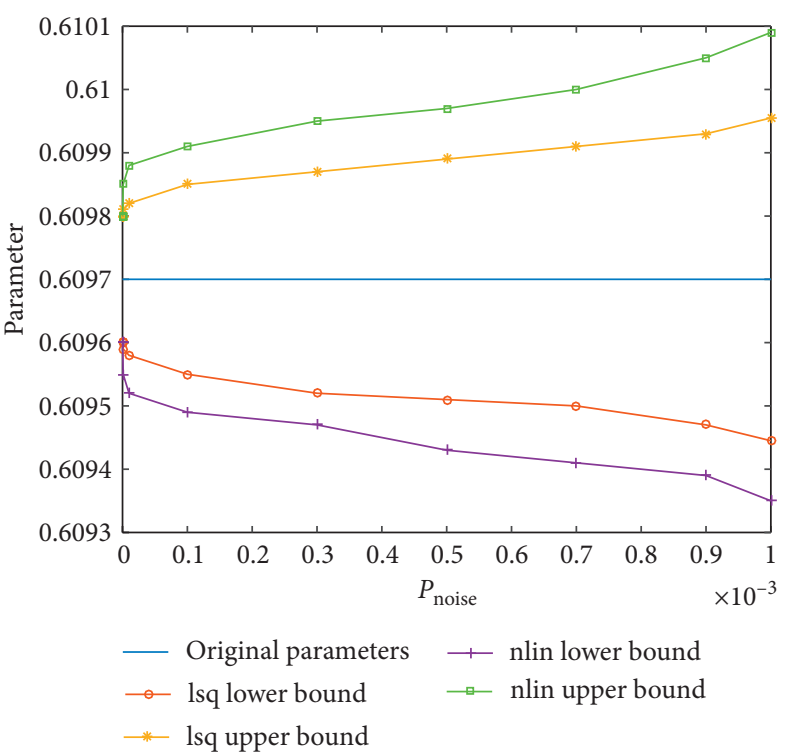

(a)

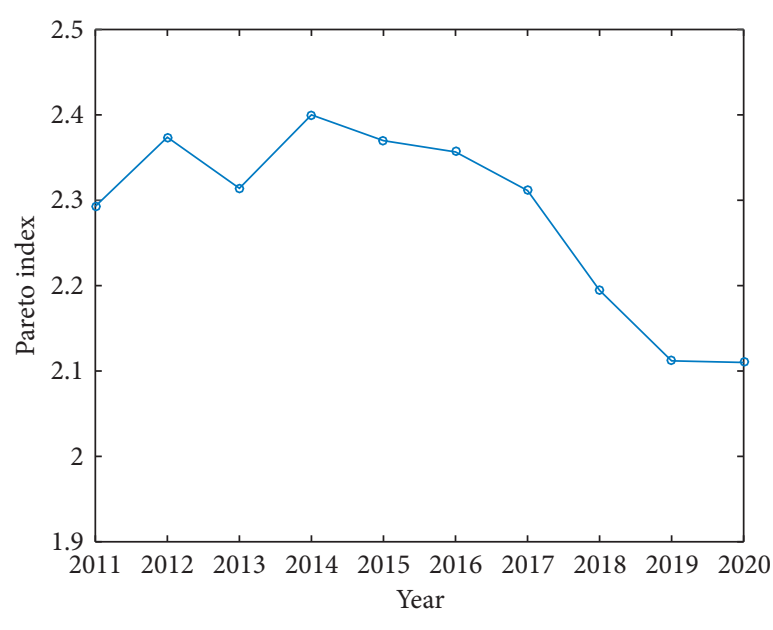

(b)

FIGURE 5: The distinguishing criterion in SVM classification; $\alpha=0.1, \beta=0.9$. (a) The upper and lower bounds of lsqcurvefit and $n l i n f i t$ under different $P_{\text {noise }}$ (b) The Pareto curve computed by lsqcurvefit algorithm.

globalization. The widening gap between the rich and the poor is inextricably linked to knowledge and education. Antipoverty is a war-preparation problem that all countries attach great importance to. The results show the rationality and effectiveness of the parameter resolution in practical application.

\section{Conclusion}

In this paper, we investigate parameter resolution systematically and present a framework to compute the parameter resolution. Parameter resolution is the natural extension of the resolution concept; we take the power law model as an example to explain the rationality, feasibility, and effectiveness of the concept of parameter resolution. Our framework can be used in different models and algorithms, even for nonGaussian noises. For power law model, the influence of each factor on the resolution system is analyzed. With the increase of the SNR, the parameter resolution is finer. Parameter resolution can be calculated in every case for any algorithm, as well as distinguishing criterion and noise, and so forth. If the estimations are asymptotically normal, parameter resolution can be approximated by interval estimation in statistics. With the decrease of significance level, the experimental slope and the theoretical slope both decrease, and the result tends to be consistent when $\alpha$ approaches zero. It can be used to select a more precise algorithm for experimental data. We use Forbes rich list as a prototype to show how to apply the parameter resolution to real data. All computation codes and data are available upon request.

\section{Data Availability}

The data used to support the findings of this study are available from the corresponding author upon request.

\section{Conflicts of Interest}

The authors declare that there are no conflicts of interest.

\section{Acknowledgments}

This work was supported by the National Key Research and Development Program of China (no. 2020YFA0714200).

\section{References}

[1] X. L. Yang, X. Wei, and Z. K. Sun, "Estimating model parameters in nonautonomous chaotic systems using synchronization," Physics Letters A, vol. 364, pp. 378-388, 2007.

[2] Kamar, S. Hashim, and B. S. Msallam, "Comparative study between generalized maximum entropy and Bayes methods to estimate the four parameter weibull growth model," Journal of Probability and Statistics, vol. 2020, Article ID 7967345, 7 pages, 2020.

[3] J. H. Ma and Y. Liu, "Exact solutions for a generalized nonlinear fractional Fokker-Planck equation," Nonlinear Analysis Real World Applications, vol. 11, pp. 515-521, 2010.

[4] D. Brkic and Z. Cojbasic, "Intelligent flow friction estimation," Computational Intelligence and Neuroscience, vol. 201610 pages, 2016.

[5] L. Wu, F. Chen, C. Y. Huang, G. H. Ding, and Y. M. Ding, "Multi-index inversion of NMR $\mathrm{t} 2$ spectra based on improved nonlinear fitting," Acta Physica Sinica, vol. 65, 2016.

[6] L. Xie, J. H. Ma, and M. Goh, "Supply chain coordination in the presence of uncertain yield and demand," International Journal of Production Research, vol. 202017 pages, 2020.

[7] T. Zhao, L. Wu, D. Li et al., "Multifractal analysis of hydrologic data using wavelet methods and fluctuation analysis," Discrete Dynamics in Nature and Society, vol. 201718 pages, 2017. 
[8] P. F. Brown, S. A. D. Pietra, and V. D. J. Pietra, "The mathematics of statistical machine translation: parameter estimation," Computational Lingus, vol. 19, pp. 263-311, 1993.

[9] H. Li, "A statistical framework for SNP calling, mutation discovery, association mapping and population genetical parameter estimation from sequencing data," Bioinformatics, vol. 27, pp. 2987-2993, 2011.

[10] L. M. Madsen, G. Fiandaca, E. Auken, and A. V. Christiansen, "Time domain induced polarization an analysis of Cole-Cole parameter resolution and correlation using Markov chain Monte Carlo inversion," Geophysical Journal International, vol. 211, pp. 1341-1353, 2017.

[11] J. Breebaart, F. Nater, and A. Kohlrausch, "Spectral and spatial parameter resolution requirements for parametric, filterbank-based HRTF processing," Journal of the Audio Engineering Society, vol. 58, pp. 126-140, 2010.

[12] N. N. Lu and J. H. Yu, "Research on resolution based on EM algorithm," Acta Mathematica Scientia (A), vol. 638-648, pp. 638-648, 2019.

[13] J. Zhang and J. H. Yu, "Resolution of linear regression model parameter estimation method," Acta Mathematica Scientia (A), vol. 40, pp. 263-274, 2020.

[14] M. Marsili and Y.-C. Zhang, "Probabilistic fragmentation and effective power law," Physical Review Letters, vol. 77, p. 3577, 1966.

[15] M. E. J. Newman, "Power laws, Pareto distributions and Zipf's law," Contemporary Physics, vol. 46, pp. 323-351, 2005.

[16] J. H. Zhang, Q. H. Chen, and Y. G. Wang, "Zipf distribution in top Chinese firms and an economic explanation," Physica A: Statistical Mechanics and Its Applications, vol. 388, pp. 2020-2024, 2009.

[17] B. S. Xu, J. Z. Guo, N. Xi, Q. H. Chen, and Y. G. Wang, "World income distribution and mobility," Physics Procedia, vol. 3, pp. 1713-1723, 2010.

[18] Y. J. Wu, J. Z. Guo, Q. H. Chen, and Y. G. Wang, "Socioeconomic implications of donation distributions," Physica A Statistical Mechanics \& Its Applications, vol. 390, pp. 43254331, 2011.

[19] Q. H. Chen, J. Z. Guo, and Y. F. Liu, "A statistical study on Chinese word and character usage in literatures from the tang dynasty to the present," Journal of Quantitative Linguistics, vol. 19, pp. 232-248, 2012.

[20] B. Gutenberg and C. F. Richter, "Frequency of earthquakes in California," Bulletin of the Seismological society of America, vol. 34, pp. 185-188, 1944.

[21] E. T. Lu and R. J. Hamilton, "Avalanches and the distribution of solar flares," The Astrophysical Journal, vol. 380, pp. L89L92, 1991.

[22] G. Neukum and B. A. Ivanov, "Crater size distributions and impact probabilities on Earth from lunar, terrestrial-planet, and asteroid cratering data," Hazards due to Comets and Asteroids, vol. 1, 1994.

[23] D. C. Roberts and D. L. Turcotte, "Fractality and self-organized criticality of wars," Fractals, vol. 6, pp. 351-357, 1998.

[24] Y.-P. Jeon and B. J. McCoy, "Evolution of power law distributions in science and society," Physical Review E, vol. 72, Article ID 37104, 2005.

[25] E. Milotti, "Exact numerical simulation of power-law noises," Physical Review E, vol. 72, Article ID 56701, 2005.

[26] S. Maslov, "Power laws in chess," Physics, vol. 2, p. 97, 2009.

[27] F. Lucchin and F. Matarrese, "Power-law inflation," Physical Review D, vol. 32, p. 1316, 1985.
[28] M. S. Mega, P. Allegrini, P. Grigolini et al., "Power-law time distribution of large earthquakes," Physical Review Letters, vol. 90, Article ID 188501, 2003.

[29] H. Takayasu, A. Provata, and M. Takayasu, "Stability and relaxation of power-law distribution," Physical Review A, vol. 42, p. 7087, 1990.

[30] C.-H. Lam and L. M. Sander, "Surface growth with power-law noise," Physical Review Letters, vol. 69, p. 3338, 1992.

[31] A. Rodriguez and A. Laio, "Clustering by fast search and find of density peaks," Science, vol. 344, p. 1492, 2014.

[32] Y. P. Chen and Q. H. Chen, "Parameter estimation of power law distribution of continuous type," Journal of Chengdu Normal University, vol. 28, pp. 113-116, 2012.

[33] X. Z. Wang, Theory and Application of Nonlinear Model Parameter Estimation, Wuhan University Press, Wuhan, China, 2002.

[34] G. Qin and J. H. Ma, "Chaos and Hopf bifurcation of a finance system," Nonlinear Dynamics, vol. 58, no. 1-2, p. 209, 2009.

[35] J. H. Ma and L. Xie, "The comparison and complex analysis on dual-channel supply chain under different channel power structures and uncertain demand," Nonlinear Dynamics, vol. 83, no. 3, pp. 1379-1393, 2016.

[36] M. Levy and S. Solomon, "New evidence for the power-law distribution of wealth," Physica A Statistical Mechanics \& Its Applications, vol. 242, pp. 90-94, 1997.

[37] S. Sinha, "Evidence for power-law tail of the wealth distribution in India," Physica A Statistical Mechanics \& Its Applications, vol. 359, pp. 555-562, 2005.

[38] M. Patriarca, A. Chakraborti, and G. Germano, "Influence of saving propensity on the power-law tail of the wealth distribution," Physica A Statistical Mechanics \& Its Applications, vol. 369, pp. 723-736, 2005.

[39] O. S. Klass, O. Biham, M. Levy, O. Malcai, and S. Solomon, "The Forbes 400 and the Pareto wealth distribution," Economics Letters, vol. 90, pp. 290-295, 2006. 